\title{
Electrical spin injection in silicon and the role of defects
}

\author{
F. Rortais, ${ }^{1,2}$ C. Vergnaud, ${ }^{1,2}$ C. Ducruet,${ }^{3}$ C. Beigné,,${ }^{1,2}$ A. Marty, ${ }^{1,2}$ J.-P. Attané, ${ }^{1,2}$ J. Widiez,${ }^{4}$ H. Jaffrès, ${ }^{5}$ \\ J.-M. George, ${ }^{5}$ and M. Jamet ${ }^{1,2}$ \\ ${ }^{1}$ Univ. Grenoble Alpes, INAC, F-38000 Grenoble, France \\ ${ }^{2}$ CEA, INAC, F-38000 Grenoble, France \\ ${ }^{3}$ CROCUS-Technology, F-38025 Grenoble, France \\ ${ }^{4}$ CEA, LETI, MINATEC Campus, F-38054 Grenoble, France \\ ${ }^{5}$ Unité Mixte de Physique, CNRS, Thales, Univ. Paris-Sud, Université Paris-Saclay, 91767 Palaiseau, France
}

(Received 4 July 2016; revised manuscript received 21 October 2016; published 16 November 2016)

\begin{abstract}
Three-terminal devices, where the same ferromagnetic electrode is used for electrical spin injection and detection, is a very easy and powerful tool to probe the spin properties in nonmagnetic materials. For instance, it has been intensively used to study spin injection and detection in silicon. However the interpretation of the magnetoresistance signals observed experimentally is still under debate. In particular, a controversy has been raised about the experimental spin signal which is orders of magnitude larger than the predicted value. Recently, Song et al. [Phys. Rev. Lett. 113, 047205 (2014)] proposed that the magnetoresistance signal measured using the Hanle effect in a three-terminal geometry is due to defects or impurities in the tunnel barrier separating the ferromagnetic electrode from the silicon channel. It has also been supported by the experimental work of Txoperena et al. [Phys. Rev. Lett. 113, 146601 (2014)]. In this study, we perform electrical spin injection/detection measurements using three-terminal devices in different silicon films and study the role of defects. For this purpose, we use the tunneling inelastic spectroscopy to measure the Hanle effect and control the presence of defects in the tunnel barrier. Contrary to previous reports, we demonstrate that defects have no significant contribution to the spin signal. From a comparison with capacitance-voltage measurements in $n$-doped germanium in which interface states contribute to the spin signal, we also conclude on the presence of interface states in silicon.
\end{abstract}

DOI: 10.1103/PhysRevB.94.174426

The concern about solutions for more than Moore electronics has become a central issue. In recent years, much research has focused on the promising solution of semiconductor (SC) spintronics. Three main technological steps are required to achieve spin electronic devices in semiconductors: spin injection, manipulation, and detection in the SC channel. Spin injection and detection could be validated using fourterminal devices in a nonlocal geometry [1-4]. In this measurement scheme, spin injection and detection are independently achieved by using two different ferromagnetic electrodes. By this, only the spin current diffusing in the SC channel from one ferromagnetic electrode to the other is detected. However, because of the difficult fabrication of such nanometer-scale devices with well-defined tunneling contacts, an alternative approach was proposed using a single ferromagnetic electrode for spin injection and spin detection. Several groups have used this three-terminal Hanle measurement [5] to study the spin properties in different nonmagnetic materials [6-15]. Unfortunately, using the same ferromagnetic electrode for spin injection and detection makes this three-terminal technique sensitive to spurious tunneling magnetoresistance effects. It can lead to measured spin signals orders of magnitude larger than the value predicted by the spin diffusion model. Different explanations were proposed without reaching a consensus in the scientific community. Some groups suggested that impurities in the tunnel barrier can give a magnetoresistance (MR) signal even in the absence of ferromagnetic electrode [16-20]. Others suggested that the spin diffusion model is no more applicable in the case of three-terminal Hanle measurements [21]. One competing model suggests that a paramagnetic layer at the interface between the ferromagnet (FM) and the tunnel barrier could be at the origin of the magnetoresistance signal [22]. Moreover, Yamamoto et al. proposed that the tunneling rate depends on the applied external field [23]. Finally some groups demonstrated that interface states confined between the tunnel barrier and the SC can play a key role in the spin signal amplification [12,24].

In this study, we use three-terminal silicon devices to discuss about the spin signal amplification. By using inelastic tunneling spectroscopy, we succeed in showing experimentally that localized defects in the tunnel barrier cannot be at the origin of the Hanle signal and spin signal amplification. We rather demonstrate that the spin signal amplification comes from sequential tunneling through confined interface states in agreement with capacitance-voltage results.

\section{SAMPLE GROWTH AND EXPERIMENTAL METHODS}

In order to understand the origin of spin signal amplification in silicon, we study electrical spin injection and detection in $n$-doped and $p$-doped silicon films on an insulator. The siliconon-insulator (SOI) wafers are made following the $\mathrm{SmartCut}^{\mathrm{TM}}$ process and consist, from bottom to top, of a silicon substrate lightly $p$ doped with boron $\left(10^{11}-10^{12} \mathrm{~cm}^{-3}\right)$, a $1-\mu$ m-thick $\mathrm{SiO}_{2}$ buried oxide layer (BOX) and an active top layer with variable thickness and doping. One active layer is implanted with phosphorous to obtain a $n$-type doping and the other one is implanted with boron to obtain a $p$-type doping. The thickness of the $n$-doped film (resp. $p$-doped film) is $215 \mathrm{~nm}$ (resp. $70 \mathrm{~nm}$ ). The doping levels and mobilities are determined using double-Hall crosses: $n=2.9 \times 10^{19} \mathrm{~cm}^{-3}, \mu=95 \mathrm{~cm}^{2} /(\mathrm{V} \mathrm{s})$ for the $n$-doped film and $p=1.3 \times 10^{19} \mathrm{~cm}^{-3}, \mu=$ $42 \mathrm{~cm}^{2} /(\mathrm{V} \mathrm{s})$ for the $p$-doped film. Such high doping levels are used to decrease the width of the Schottky barrier in silicon. 


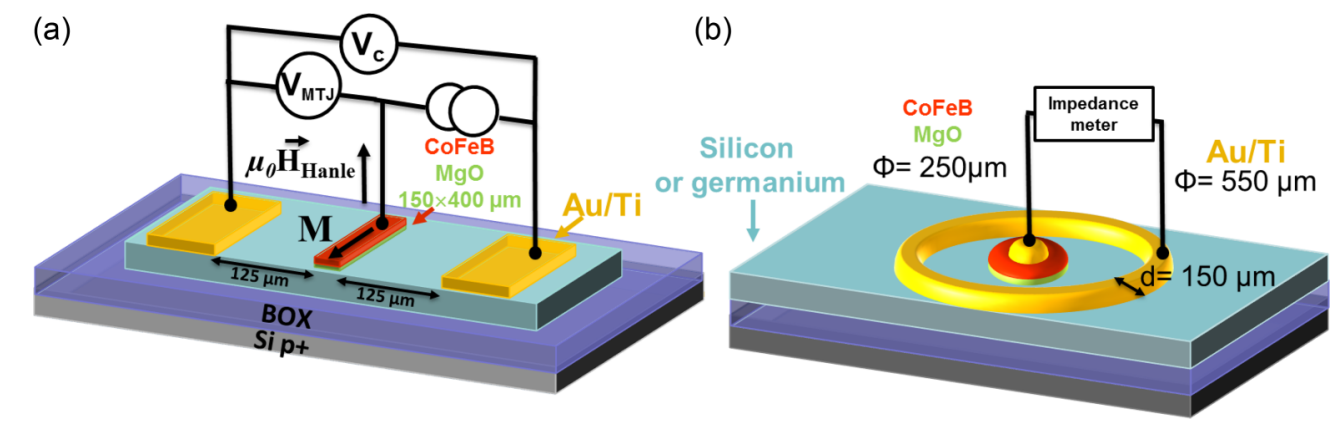

(b)

FIG. 1. (a) Sketch of the three-terminal device used for electrical spin injection and detection by Hanle effect measurements. The top silicon layer is either $n$-doped or $p$-doped and the same ferromagnetic electrode $(\mathrm{Ta} / \mathrm{CoFeB} / \mathrm{MgO})$ is used for the electrical spin injection and detection. The same device is used to investigate the presence of defects in the tunnel barrier by inelastic tunneling spectroscopy. (b) Sketch of the device used for capacitance-voltage measurements to probe the presence of the interface state.

A ferromagnetic tunnel junction $[\mathrm{Ta}(5 \mathrm{~nm}) / \mathrm{CoFeB}(5$ $\mathrm{nm}) / \mathrm{MgO}(3.3 \mathrm{~nm})]$ is used as an electrical spin injector to spin polarize the charge current as sketched in Fig. 1(a). In order to grow this stack, the 10-nm-thick $\mathrm{SiO}_{2}$ capping layer on top of the SOI wafer is removed using hydrofluoric acid and the sample is transferred to the growth chamber in deionized water. Then, using conventional DC magnetron sputtering at a base pressure of $7 \times 10^{-9}$ mbar, a 1.1-nm-thick Mg layer is deposited at a rate of $0.02 \mathrm{~nm} \mathrm{~s}^{-1}$ and an argon pressure of $2 \times 10^{-3} \mathrm{mbar}$ at room temperature. It is immediately followed by plasma oxidation during $30 \mathrm{~s}$ at an oxygen pressure of $6 \times 10^{-3}$ mbar and a radio-frequency power of $100 \mathrm{~W}$. This cycle is repeated three times to finally obtain a 3.3-nm-thick $\mathrm{MgO}$ tunnel barrier. The 5-nm-thick $\mathrm{Co}_{60} \mathrm{Fe}_{20} \mathrm{~B}_{20}$ ferromagnetic layer is deposited using DC magnetron sputtering at a $0.03 \mathrm{~nm} \mathrm{~s}^{-1}$ deposition rate and capped with a 5-nm-thick Ta layer to prevent oxidation. The full stack is annealed at $300{ }^{\circ} \mathrm{C}$ during 90 minutes. Conventional optical lithography is then used to define three-terminal devices as shown in Fig. 1 corresponding to a central ferromagnetic tunnel junction $\left(150 \times 400 \mu \mathrm{m}^{2}\right)$ in between two ohmic contacts $\left(150 \times 500 \mu \mathrm{m}^{2}\right)$.

The spin accumulation generated by electrical injection is detected using the Hanle effect as shown in Fig. 2. For this purpose, a DC current $I$ is applied between the ferromagnetic electrode and one ohmic contact while the voltage $V_{M T J}$ is recorded between the ferromagnetic electrode and the second ohmic contact. These parameters are defined in Fig. 1(a). $V_{M T J}$ can be written as $V_{M T J}=V_{M T J}^{0}+V_{M T J}(H) ; V_{M T J}^{0}$ is the constant offset voltage due to the current flowing through the $\mathrm{MTJ} / \mathrm{silicon}$ interface. In the following, $V_{M T J}^{0}$ is called the bias voltage applied to the MTJ. $V_{M T J}(H)$ is the magnetic field dependent part which contains the spin accumulation signal. A magnetic field is applied perpendicular to the silicon film in order to induce spin precession. As a result of spin precession, $V_{M T J}(H)$ decreases with the applied magnetic field by $\Delta V_{\perp}$ $(>0)$ following a Lorentzian curve which is the signature of the Hanle effect. By further increasing the applied magnetic field, the magnetization of the ferromagnetic electrode starts rotating out-of-plane. At this stage, $V_{M T J}(H)$ increases because the injected spins align along the external magnetic field which progressively suppresses the Larmor precession.

However, the Hanle effect measurements may be parasited by spurious tunneling magnetoresistance effects. Moreover, spin dephasing due to random stray fields created at rough interfaces reduces the spin signal even in zero external field. This effect can be quantified by measuring the so-called inverted Hanle effect, i.e., $V_{M T J}$ as a function of the magnetic field applied in-plane along the electrode magnetization. When the applied field becomes larger than the random stray fields, the spin accumulation is restored and the field dependent part of the measured voltage $\left(V_{M T J}(H)\right)$ increases by $\Delta V_{/ /}(>0)$ following a reversed Lorentzian curve as shown in Fig. 2. The total spin signal $\Delta V$ is then given by the sum of the Hanle and inverted Hanle effects: $\Delta V=\Delta V_{/ /}+\Delta V_{\perp}$. In the following, the spin signal is rather expressed as the $\operatorname{spin} R A$ product given by: $\operatorname{spin} R A=(\Delta V / I) \times A$ where $I$ and $A$ are the applied DC current and ferromagnetic electrode area, respectively. The temperature dependence of the spin signal is studied carefully from $2 \mathrm{~K}$ up to room temperature using an Oxford Spectromag cryostat. The DC current $I$ is applied between the ferromagnetic tunnel junction and one ohmic contact in order to work at fixed bias voltage $V_{M T J}^{0} . V_{M T J}$ and (a)

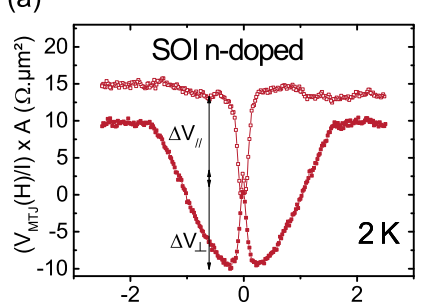

(c)

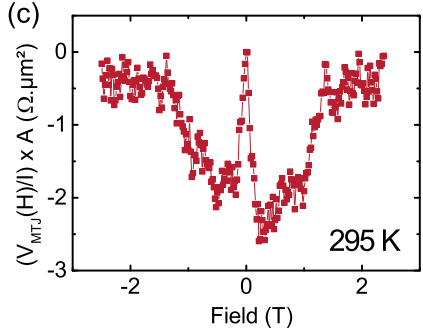

(b)

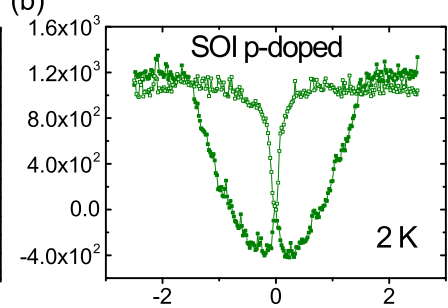

(d)

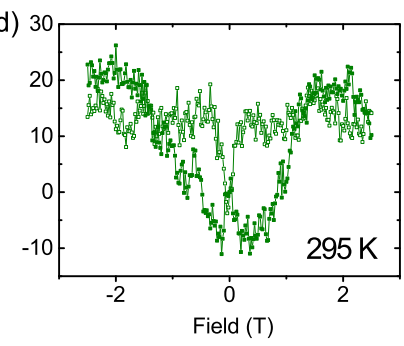

FIG. 2. Direct Hanle effect (full symbols) and inverted Hanle effect (open symbols) in $n$-doped silicon (red symbols) and (b) $p$-doped silicon (green symbols). (a),(b) $T=2 \mathrm{~K}$ and (c),(d) $T=$ $295 \mathrm{~K} . V_{M T J}(H)$ is defined as $V_{M T J}-V_{M T J}^{0}$ (see text). The bias voltages applied on the magnetic tunnel junctions are $0.4 \mathrm{~V}$ in $n$-doped $\mathrm{Si}$ and $0.17 \mathrm{~V}$ in $p$-doped $\mathrm{Si}$, respectively. 
$V_{C}$ as defined in Fig. 1(a) are recorded using nanovoltmeters, and the measurements are reproduced on several devices.

To test whether the spin signal amplification is due to defects or impurities in the tunnel barrier, we use inelastic tunneling spectroscopy (IETS) measurements. Tunneling processes through defects in the tunnel barrier are most probably inelastic, and they can be detected using this technique [25]. Inelastic tunneling processes lead to slope changes at given bias voltages in the $I\left(V_{M T J}\right)$ curves of the magnetic tunnel junction, but they are more visible as peaks in the second derivative of $I\left(V_{M T J}\right)$ curves [25,26]. An AC current source (working at $931 \mathrm{~Hz}$ ) and a lock-in amplifier are combined to record the first and second harmonics of the voltage measured on the tunnel junction. Meanwhile, a DC current offset is applied to modify the bias voltage on the tunnel junction. Finally $I\left(V_{M T J}\right)$ curves are measured in static conditions using a DC current source and a nanovoltmeter. The voltage dependence can be found from the current dependence using the following expression [25]:

$$
\frac{d^{2} I}{d V_{M T J}^{2}}=-\left(\frac{d I}{d V_{M T J}}\right)^{3} \frac{d^{2} V_{M T J}}{d I^{2}} .
$$

Because interface states can be at the origin of the spin signal amplification, a comparison with $n$-doped germanium was used. Indeed, in $n$-doped Ge, we have already demonstrated the presence of interface states between $\mathrm{MgO}$ and $\mathrm{Ge}$ [10]. We also carried out preliminary capacitance-voltage $\left[C\left(V_{M T J}\right)\right]$ measurements as a function of temperature. Interface states are identified in $C\left(V_{M T J}\right)$ curves as peaks close to zero bias, which is dependent on the frequency and the temperature. They give a visible signal at low frequency but they are no more contributing to the capacitance at high frequencies. We use the same samples as the ones for three-terminal measurements and the $C\left(V_{M T J}\right)$ measurements scheme, shown in Fig. 1(b), is processed on top by optical lithography. They are then glued on ceramics and the $C\left(V_{M T J}\right)$ measurements are conducted using a probe station and an Agilent E4980A impedancemeter. The temperature is varied from $4 \mathrm{~K}$ to room temperature. The bias voltage applied to the tunnel junction is varied from zero up to $2 \mathrm{~V}$ and the frequency from $1 \mathrm{kHz}$ to $1 \mathrm{MHz}$.

\section{RESULTS}

As mentioned in the introduction, the experimental spin signal deduced from three-terminal Hanle measurements is much larger than the value predicted by the spin diffusion model (see Table I). One possible explanation is the presence of spurious tunneling magnetoresistance effects. In order to test the validity of this explanation, we have measured the spin signals in $n$ and $p$-doped silicon films using the three-terminal Hanle measurements at various temperature and bias voltages. As shown in Fig. 2, we measure clear Hanle and inverted Hanle signals at $2 \mathrm{~K}$ and room temperature in $n$-doped and $p$-doped silicon films. The same measurements performed on a reference sample $\mathrm{Cu} / \mathrm{MgO} / \mathrm{Si}$ give no signal. We fit the experimental Hanle signal using the following Lorentzian expression:

$$
\Delta V=\frac{\Delta V_{0}}{\left(1+\left(\omega_{L} \tau_{s f}\right)^{2}\right)},
$$

where $\tau_{s f}$ is the spin lifetime and $\omega_{L}$ is the Larmor frequency $\omega_{L}=\frac{g \mu_{B} B_{z}}{\hbar} . g$ is the Landé factor and $\mu_{B}$ is the Bohr magneton. Considering the dispersion in the values of the $g$ factors in $p$-type Si [27,28] and its strong anisotropy, we decided to use $g=2$ as an average value. In the following, we assume that spin injection takes place into the silicon channel.

The spin lifetimes extracted from the width of the Hanle curves are of the order of 100 ps in $n$-doped silicon and $80 \mathrm{ps}$ in $p$-doped silicon at $2 \mathrm{~K}$. These values are in good agreement with previous measurements using the Hanle effect in three-terminal devices $[6,8,29,30]$ but much less than the ones obtained by four-terminal nonlocal measurements [3133]. We then calculate the diffusion coefficient $D$ to deduce the spin diffusion length from the spin lifetime. $D$ is defined as:

$$
\frac{D}{\mu}=\frac{k_{B} T}{e} \frac{F_{1 / 2}(\eta)}{F_{-1 / 2}(\eta)},
$$

where $\mu$ is the carrier mobility measured experimentally. $k_{B}$ is the Boltzmann constant, $T$ the temperature, and $e$ the elementary electron charge. $F_{1 / 2}$ and $F_{-1 / 2}$ are the Fermi-Dirac integrals defined as:

$$
F_{j}(x)=\frac{1}{\Gamma(j+1)} \int_{0}^{\infty} \frac{t^{j}}{1+\exp (t-x)} d t .
$$

After developing the Fermi-Dirac integrals, one obtains:

$$
D=\frac{2}{3} \mu \frac{E_{f}}{e}\left(1+\frac{\pi^{2}}{6 \eta^{2}}\right),
$$

where $\quad E_{f}=E_{f}(0)\left(1-\frac{k_{B}^{2} T^{2} \pi^{2}}{12 E_{f}(0)^{2}}\right) \quad$ and $\quad E_{f}(0)=\frac{h^{2}}{2 \pi m^{*}}$ $\left(\frac{3 \sqrt{\pi} N}{8}\right)^{2 / 3} ; \eta=\frac{E_{f}}{k_{B} T}$.

$N$ is carrier density. We find $D_{n-S i} \approx 7.4 \mathrm{~cm}^{2} \mathrm{~s}^{-1}$ and

\begin{tabular}{|c|c|c|c|c|c|c|}
\hline \multirow[b]{3}{*}{ Silicon film } & \multirow{2}{*}{\multicolumn{2}{|c|}{$\frac{\operatorname{Spin} R A_{\text {theo }}}{\left(\Omega \mu \mathrm{m}^{2}\right)}$}} & \multicolumn{2}{|c|}{$\operatorname{Spin} R A_{\exp }$} & \multirow{2}{*}{\multicolumn{2}{|c|}{$\operatorname{Spin} R A_{\text {exp }} / \operatorname{Spin} R A_{\text {theo }}$}} \\
\hline & & & & & & \\
\hline & LT & RT & LT & RT & LT & RT \\
\hline$n$-doped & 130 & 15 & $25 \times 10^{3}$ & $2.5 \times 10^{3}$ & $\approx 200$ & $\approx 160$ \\
\hline$p$-doped & 53 & 6.4 & $1.6 \times 10^{6}$ & $30 \times 10^{3}$ & $\approx 30000$ & $\approx 5000$ \\
\hline
\end{tabular}
$D_{p-S i} \approx 0.83 \mathrm{~cm}^{2} \mathrm{~s}^{-1}$ at $2 \mathrm{~K}$. Using these values and the

TABLE I. Comparison between experimental and expected spin signals at low (LT) and room (RT) temperature. The spin lifetimes to calculate $\operatorname{spin} R A_{\text {theo }}$ are taken from Refs. $[30,35,36]$. In the absence of data for the spin lifetime in $p$-doped Si at low temperature, we used ten times the spin lifetime at room temperature by analogy with $n$-doped $\mathrm{Si}$. 

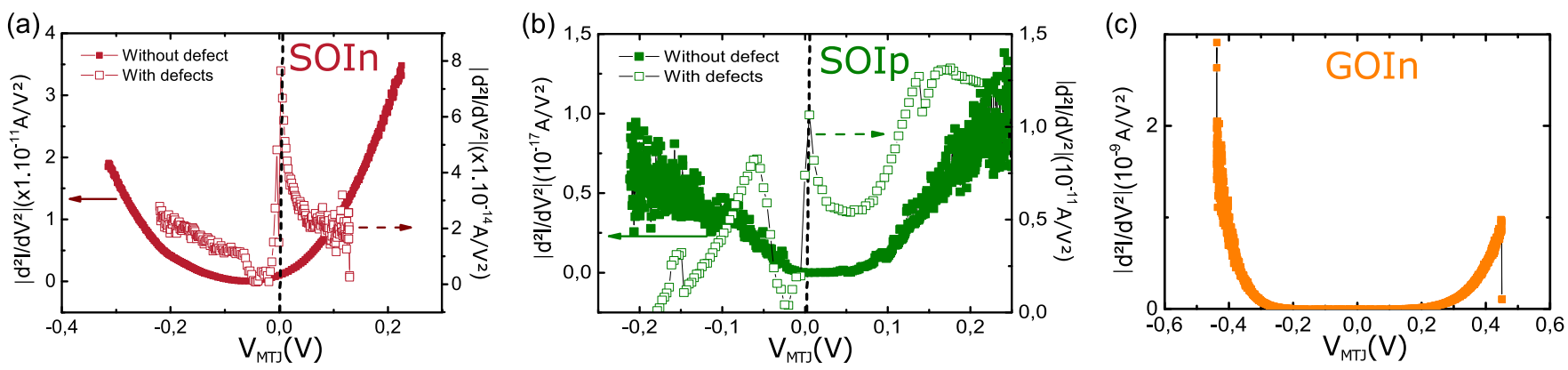

FIG. 3. (a) Inelastic tunneling spectroscopy performed at $2 \mathrm{~K}$ in $n$-doped silicon and (b) in $p$-doped silicon. The creation of defects is possible by applying an electrical stress to the tunnel junction, and it leads to the appearance of additional peaks in the spectra. The results with defects (resp. without defects) in the tunnel barrier are shown with open symbols (resp. full symbols). (c) Inelastic tunneling spectroscopy recorded in $n$-doped germanium at $2 \mathrm{~K}$.

experimental spin lifetimes, we obtain the spin diffusion lengths at $2 \mathrm{~K}: 300 \mathrm{~nm}$ in $n$-doped silicon and $80 \mathrm{~nm}$ in $p$-doped silicon, respectively. The inverted Hanle effect indicates the presence of magnetic stray fields at the interface between the ferromagnetic tunnel junction and the silicon film. The spins injected close to the interface are sensitive to these random fields which leads to a reduction of the spin signal under zero applied field and to a shorter spin lifetime as deduced from the Hanle curve [6]. Other authors discussed this effect in terms of internal magnetic fields at the level of defects within the tunnel barrier [19]. Concerning the amplitude of the spin signal, we first observe that it is much larger in $p$-doped silicon than in n-doped silicon (see Fig. 2).

In the standard spin diffusion model as detailed by Fert and Jaffrès in Ref. [34], one considers the ferromagnet/tunnel barrier/semiconductor interface with a spin dependent resistance: $r_{+(-)}=2 r_{b}[1-(+) \gamma] .+(-)$ refers to the absolute spin direction of the electrons, $r_{b}$ is the interface resistance, and $\gamma$ is the spin polarization of the tunnel current. Moreover, in the ferromagnet, the spin dependent resistivity writes: $\rho_{\uparrow(\downarrow)}=2[1-(+) \beta] \rho_{F}$, where $\uparrow(\downarrow)$ refers to the majority (minority) spin direction and $\beta$ to the spin polarization of the current in the ferromagnet. $\rho_{\uparrow(\downarrow)}=2 \rho$ is the spin-independent resistivity in the semiconductor. Finally, two more constants called the spin resistances have to be defined: $r_{F}=\rho_{F} \times L_{s f}^{F}$ in the ferromagnet and $r=\rho \times L_{s f}$ in the semiconductor. $L_{s f}^{F}$ and $L_{s f}$ are the spin diffusion length in the ferromagnet and in the semiconductor, respectively. In this model, $J_{+}(z)\left[J_{-}(z)\right]$ and $\mu_{+}(z)\left[\mu_{-}(z)\right]$ are the current density and electrochemical potential of the spin $+[$ spin -$]$ electrons at position $z$ (the difference between + and - comes from the spin accumulation effects). In the limit where the spin relaxation is much slower than the momentum relaxation, the three main equations of the spin diffusion model are then:

$$
\begin{gathered}
J_{+(-)}=\frac{1}{|e| \rho_{+(-)}} \frac{\partial \mu_{+(-)}}{\partial z} \\
J_{+}+J_{-}=J \\
\frac{\partial\left(J_{+}-J_{-}\right)}{\partial z}=\frac{2 e N\left(E_{F}\right) \Delta \mu}{\tau_{s f}},
\end{gathered}
$$

where $J$ is the total current density, $\tau_{s f}$ is the spin lifetime, $2 N\left(E_{F}\right)$ is the total density of states at the Fermi level, and
$\Delta \mu=\mu_{+}(z)-\mu_{-}(z)$. It leads to the spin diffusion equation:

$$
\frac{\partial^{2} \Delta \mu}{\partial z^{2}}=\frac{\Delta \mu}{L_{s f}^{2}} .
$$

This equation can be solved with the proper boundary conditions. In the case where $r_{b} \gg r_{F}, r$ corresponding to our system, one finds at the tunnel barrier/semiconductor interface: $\Delta \mu=2 \gamma \rho L_{s f} J$, where $J=I / A, I$ is the DC applied current, and $A$ the MTJ area. When the film thickness $t$ is less than the spin diffusion length $L_{s f}$, which is the case in the Si films we consider, one has to renormalize $\Delta \mu$ by $L_{s f} / t$ to take into account the vertical spin confinement effect (see Ref. [34]). In that case, $\Delta \mu=2 \gamma \rho L_{s f}\left(L_{s f} / t\right)$. Finally, in a three-terminal geometry, the same MTJ measures half the spin accumulation as a voltage: $\Delta V=\frac{\gamma \Delta \mu}{2}=\frac{\gamma^{2} \rho L_{s f}^{2}}{t} \frac{I}{A}$ and the $\operatorname{spin} R A$ product writes:

$$
\operatorname{Spin} R A=(\Delta V / I) \times A=\gamma^{2} \rho L_{s f}^{2} / t,
$$

where $\gamma$ is the spin polarization of the tunnel current $(0.56$ in this case [10]), $L_{s f}$ is the spin diffusion length, and $\rho$ and $t$ are the resistivity and thickness of the silicon channel, respectively. The resistivities are determined separately at low temperature using double Hall crosses: $\rho_{n-S i}=1.2 \times 10^{-5} \Omega \mathrm{m}$ and $\rho_{p-S i}=1.0 \times 10^{-4} \Omega \mathrm{m}$. The experimental and theoretical results are summarized in Table I.

If we assume that spin injection takes place in the $\mathrm{Si}$ channel, the difference between the experimental and theoretical values is considerable. The measured signals are orders of magnitude larger than the expected ones. Moreover, the dependence of the Hanle signal on the applied current is nonlinear while the spin diffusion model predicts a linear one. We thus investigate the possible presence of defects in the tunnel barrier by using inelastic tunneling spectroscopy on the same samples as those used for three-terminal measurements. In Figs. 3(a) and 3(b), we can see peaks close to zero bias which are characteristic of the presence of defects. In $n$-doped $\mathrm{Si}$, we could find several devices on the same chip exhibiting a low bias peak in IETS as shown in Fig. 3(a). On the other hand, in $p$-doped $\mathrm{Si}$, we had to apply an electrical stress to defect-free devices in order to observe a clear low bias peak in IETS [Fig. 3(b)]. To do so, we increased the bias voltage on the tunnel junction until the $I(V)$ curve is irreversibly modified. This method is highly efficient and reproducible 

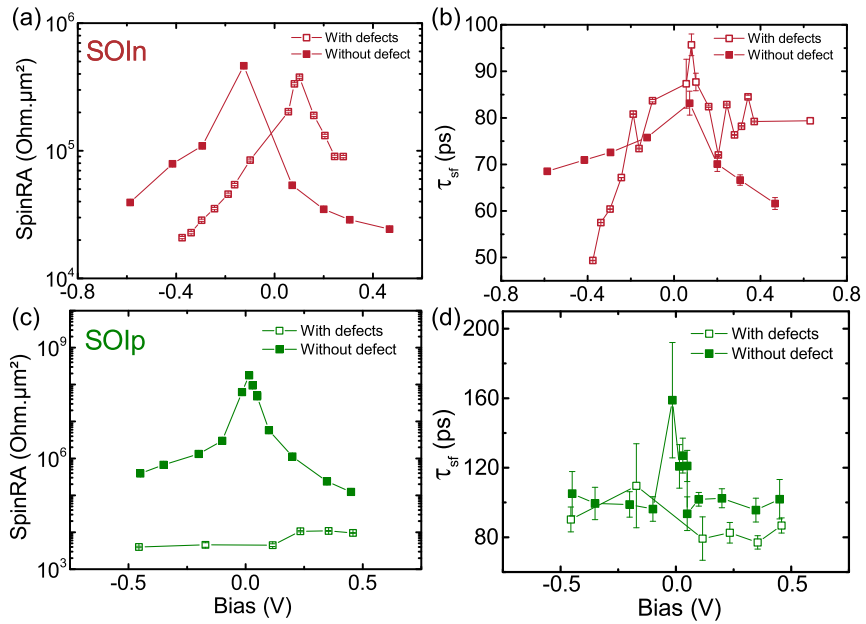

FIG. 4. Bias dependence at $2 \mathrm{~K}$ of the spin signal and spin lifetime extracted from Hanle measurements in (a), (b) $n$-doped silicon, and (c),(d) $p$-doped silicon. The results with defects (resp. without defects) in the tunnel barrier are shown with open symbols (resp. full symbols). The black vertical dashed lines indicate the bias positions at which we observe a peak in IETS measurements (see Fig. 3).

to create defects in the tunnel barrier. As a reference, IETS in $n$-doped Ge is shown in Fig. 3(c). No peak corresponding to defects in the tunnel barrier is visible in the spectrum, while the existence of localized states at the interface between $\mathrm{MgO}$ and Ge has been demonstrated experimentally [10]. We have then studied the bias dependence of the spin signal and spin lifetime with and without defects on several devices. The results are presented in Fig. 4 for both $n$-doped and $p$-doped Si. In the case of $n$-doped $\mathrm{Si}$, the spin signal remains almost unchanged: We find the same order of magnitude and the same overall bias dependence except that the maximum value is shifted towards positive bias as shown in Fig. 4(a). We can also extract very similar spin lifetimes from the Hanle effect [Fig. 4(b)]. In the case of $p$-doped $\mathrm{Si}$, the spin signal is drastically reduced after the electrical stress and the introduction of defects in the tunnel barrier as shown in Fig. 4(c). However, it still remains more than two orders of magnitude larger than the predicted value. The $R A$ product of the tunnel junction drops from $8.4 \times 10^{6} \mathrm{k} \Omega \mu \mathrm{m}^{2}$ down to $1.4 \times 10^{3} \mathrm{k} \Omega \mu \mathrm{m}^{2}$, whereas it remains almost the same in $n$-doped $\mathrm{Si}\left(\approx 1.8 \times 10^{4} \mathrm{k} \Omega \mu \mathrm{m}^{2}\right)$. This sharp decrease of the $R A$ product in $p$-doped Si makes low bias voltage measurements difficult due to the limited applied currents and poor signal-to-noise ratio. In Fig. 4(d), despite the strong reduction of the spin signal, we find spin lifetimes similar to those in defect-free devices. Finally, in both $n$-doped and $p$-doped silicon, there is no spin signal nor spin lifetime singularity at the bias position of defects indicated by vertical dashed lines in Fig. 4. In conclusion, the presence of defects in the tunnel barrier cannot be at the origin of both the spin signal magnification and low spin lifetime values in Hanle measurements.

One possible origin may be the presence of localized states at the interface between $\mathrm{MgO}$ and Si leading to a two-step tunneling process as already discussed in GaAs [24] or Ge $[7,10]$. The nature of these defects may be twofold. First, prior to the $\mathrm{MgO}$ growth, silicon surfaces were prepared using hydrofluoric acid and DI water. They are then exposed for a few minutes to air before the deposition of $\mathrm{MgO}$. At this stage, the density of surface defects is most probably much higher than the one obtained in the industry $\left(10^{10} \mathrm{~cm}^{-2}\right)$. Moreover, the growth of $\mathrm{MgO}$ is achieved in two steps: We first deposit pure $\mathrm{Mg}$ and then use an oxygen plasma to form $\mathrm{MgO}$. During this process, we have optimized the oxidation time, nevertheless we believe that the silicon surface underneath is partially oxidized. It leads to the formation of additional interface defects. The presence of interface states could explain why the experimental spin parameters do not depend on the presence of defects in the tunnel barrier. The sharp drop of the spin signal in $p$-doped $\mathrm{Si}$ after the electrical creation of defects can be explained by the poor spin injection efficiency into interface states through the damaged tunnel barrier. Moreover, when interface states are strongly confined, the electron transit time into these states becomes longer than the spin lifetime and only the spin accumulation into interface states is detected by Hanle measurements, i.e., spin accumulation in the $\mathrm{Si}$ channel is negligible. Due to their localized character, the spin resistance of interface states is much larger than in Si leading to the spin signal amplification we observe experimentally. Finally, the spin lifetime deduced from Hanle curves is too short to correspond to the intrinsic spin lifetime into interfaces states but rather reflects the spin dephasing introduced by the interface random magnetic stray fields. This is further confirmed by the low bias and temperature dependence of $\tau_{s f}$. At $2 \mathrm{~K}, \tau_{s f}$ is almost constant with the applied bias voltage as shown in Figs. 4(b) and 4(d) in $n$-doped and $p$-doped $\mathrm{Si}$, respectively. Moreover, $\tau_{s f}$ only varies from 100 ps (resp. $80 \mathrm{ps}$ ) at $2 \mathrm{~K}$ to 62 ps (resp. $85 \mathrm{ps)} \mathrm{at} \mathrm{room}$ temperature in $n$-doped $\mathrm{Si}$ (resp. $p$-doped $\mathrm{Si}$ ). In order to qualitatively support this conclusion, we have also performed $C(V)$ measurements using the device shown in Fig. 1(b). The results on defect-free $n$-doped and $p$-doped Si devices along with the $C(V)$ measurements in $n$-doped Ge, in which the presence and influence of interface states is well established, are shown in Fig. 5.

At low temperature in Figs. 5(a), 5(c), and 5(e), in all systems, we clearly see the presence of low bias peaks that disappear when increasing the excitation frequency. This observation is consistent with the presence of charge trapping into interface states [37]. At higher temperature, the electronic confinement of interface states can be thermally overcome as shown in Figs. 5(b), 5(d), and 5(f). The disappearance of the low bias peak in $n$-doped $\mathrm{Ge}$ at $175 \mathrm{~K}$ is in agreement with the transition from spin accumulation into interface states to spin injection in the Ge conduction band observed in Ref. [10]. The low bias peaks also disappear at room temperature in $n$-doped and $p$-doped Si suggesting that spin injection also takes place in the $\mathrm{Si}$ conduction band and valence band, respectively. However, the measured capacitance still depends on the frequency in both Si films. A transition in temperature is also observed in Hanle measurements as shown in Figs. 2(c) and 2(d). At room temperature, the spin signal decreases by one order of magnitude in $n$-doped $\mathrm{Si}$ and by two orders of magnitude in $p$-doped Si. However, as shown in Table I, the measured spin signal is still orders of magnitude larger than the expected one in both $n$-doped $\mathrm{Si}$ and $p$-doped $\mathrm{Si}$ at room temperature. It proves that interface states remain confined 

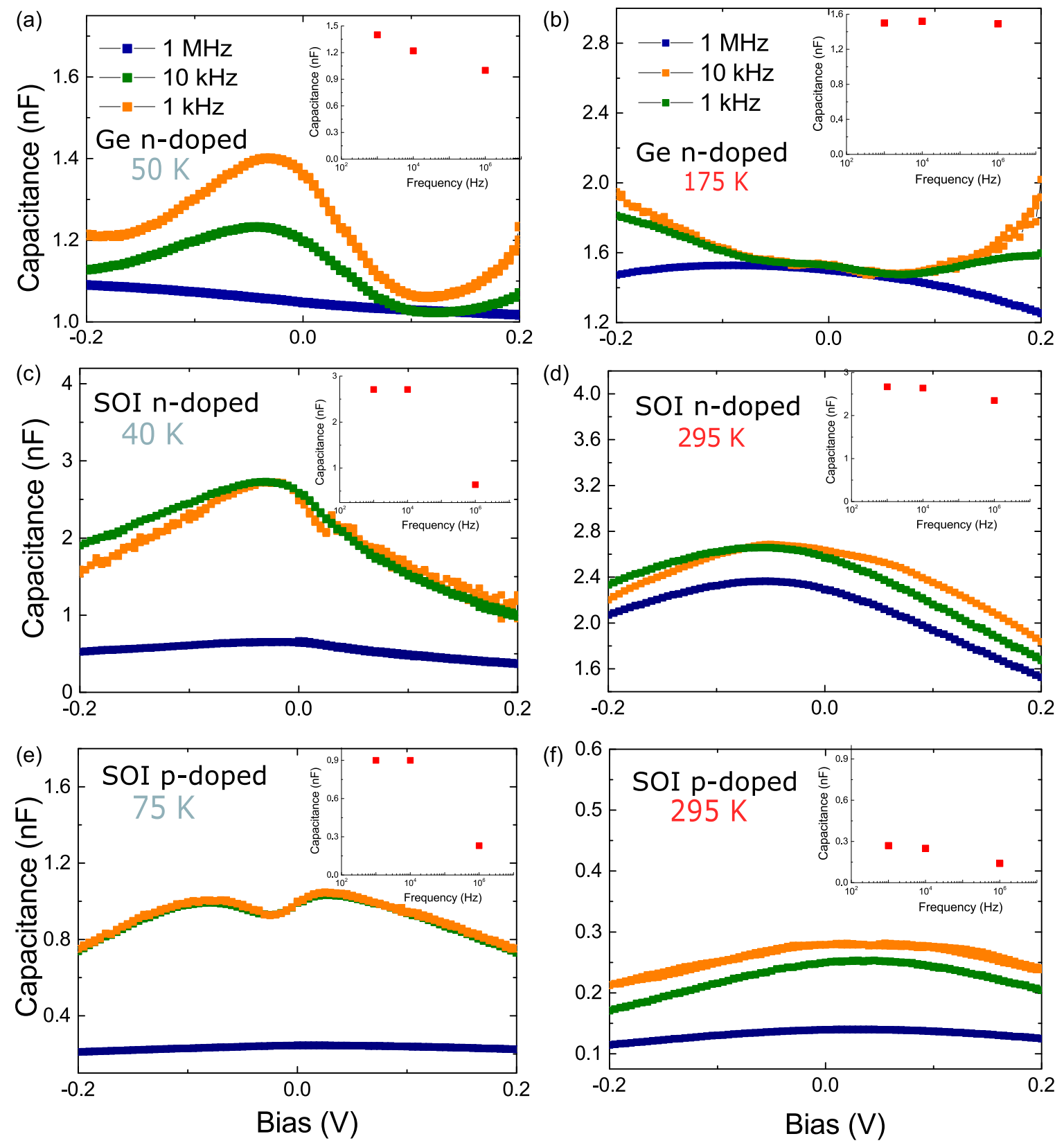

FIG. 5. Frequency dependence of capacitance-voltage curves in $n$-doped germanium at (a) $50 \mathrm{~K}$ and (b) $175 \mathrm{~K}$, in $n$-doped silicon at (c) $40 \mathrm{~K}$ and (d) $295 \mathrm{~K}$, and in $p$-doped silicon at (e) $75 \mathrm{~K}$ and (f) $295 \mathrm{~K}$. Insets show the capacitance as a function of frequency at a bias voltage of $-25 \mathrm{mV}$.

even at room temperature contrary to the case of $n$-doped germanium. It leads to two-step tunneling and spin signal amplification in the whole temperature range which is probably due to the larger band gap of Si as compared to Ge.

\section{CONCLUSION}

In summary, we have shown that defects in the tunnel barrier have little influence on Hanle measurements in silicon. For this purpose, we have fabricated three-terminal devices on $n$-doped and $p$-doped Si and used inelastic tunneling spectroscopy to control the presence of defects in the tunnel barrier. We could demonstrate that Hanle measurements are insensitive to the presence of defects. This result shows that the anomalously large spin signal magnitude is rather related to the presence of localized states at the $\mathrm{MgO} / \mathrm{Si}$ interface. This conclusion is qualitatively supported by preliminary frequency-dependent $C(V)$ measurements in which interface states are clearly present at low temperature.

\section{ACKNOWLEDGMENTS}

The authors would like to thank Léo Bonnefond and Yohan Rothman for their help in $C(V)$ measurements and Lucien Notin for his technical assistance. They also acknowledge the financial support from the French National Research Agency through the ANR project SiGeSPIN ANR-13-BS10-0002. 
[1] O. M. J. van 't Erve, A. T. Hanbicki, M. Holub, C. H. Li, C. Awo-Affouda, P. E. Thompson, and B. T. Jonker, Appl. Phys. Lett. 91 (2007).

[2] T. Suzuki, T. Sasaki, T. Oikawa, M. Shiraishi, Y. Suzuki, and K. Noguchi, Appl. Phys. Exp. 4, 023003 (2011).

[3] T. Sasaki, T. Suzuki, Y. Ando, H. Koike, T. Oikawa, Y. Suzuki, and M. Shiraishi, Appl. Phys. Lett. 104, 052404 (2014).

[4] T. Sasaki, Y. Ando, M. Kameno, T. Tahara, H. Koike, T. Oikawa, T. Suzuki, and M. Shiraishi, Phys. Rev. Appl. 2, 034005 (2014).

[5] O. Txoperena and F. Casanova, J. Phys. D 49, 133001 (2016).

[6] S. P. Dash, S. Sharma, R. S. Patel, M. P. de Jong, and R. Jansen, Nature (London) 462, 491 (2009).

[7] A. Jain, L. Louahadj, J. Peiro, J. Le Breton, C. Vergnaud, A. Barski, C. Beigné, L. Notin, A. Marty, V. Baltz et al., Appl. Phys. Lett. 99, 162102 (2011).

[8] K.-R. Jeon, B.-C. Min, I.-J. Shin, C.-Y. Park, H.-S. Lee, Y.-H. Jo, and S.-C. Shin, Appl. Phys. Lett. 98, 262102 (2011).

[9] C. H. Li, O. M. J. Van't Erve, and B. T. Jonker, Nat. Commun. 2, 245 (2011).

[10] A. Jain, J.-C. Rojas-Sanchez, M. Cubukcu, J. Peiro, J. C. Le Breton, E. Prestat, C. Vergnaud, L. Louahadj, C. Portemont, C. Ducruet, V. Baltz, A. Barski, P. Bayle-Guillemaud, L. Vila, J.-P. Attané, E. Augendre, G. Desfonds, S. Gambarelli, H. Jaffrès, J.-M. George, and M. Jamet, Phys. Rev. Lett. 109, 106603 (2012).

[11] S. Iba, H. Saito, A. Spiesser, S. Watanabe, R. Jansen, S. Yuasa, and K. Ando, Appl. Phys. Exp. 5, 053004 (2012).

[12] A. Jain, C. Vergnaud, J. Peiro, J. C. Le Breton, E. Prestat, L. Louahadj, C. Portemont, C. Ducruet, V. Baltz, A. Marty, A. Barski, P. Bayle-Guillemaud, L. Vila, J. P. Attané, E. Augendre, H. Jaffrès, J.-M. George, and M. Jamet, Appl. Phys. Lett. 101, 022402 (2012).

[13] A. Dankert, R. S. Dulal, and S. P. Dash, Sci. Rep. 3, 3196 (2013).

[14] M. Ishikawa, H. Sugiyama, T. Inokuchi, T. Tanamoto, K. Hamaya, N. Tezuka, and Y. Saito, J. Appl. Phys. 114, 243904 (2013).

[15] S. Sharma, A. Spiesser, S. P. Dash, S. Iba, S. Watanabe, B. J. van Wees, H. Saito, S. Yuasa, and R. Jansen, Phys. Rev. B 89, 075301 (2014).

[16] O. Txoperena, M. Gobbi, A. Bedoya-Pinto, F. Golmar, X. Sun, L. E. Hueso, and F. Casanova, Appl. Phys. Lett. 102, 192406 (2013).

[17] H. N. Tinkey, P. Li, and I. Appelbaum, Appl. Phys. Lett. 104, 232410 (2014).
[18] Z. Yue, M. C. Prestgard, A. Tiwari, and M. E. Raikh, Phys. Rev. B 91, 195316 (2015).

[19] Y. Song and H. Dery, Phys. Rev. Lett. 113, 047205 (2014).

[20] O. Txoperena, Y. Song, L. Qing, M. Gobbi, L. E. Hueso, H. Dery, and F. Casanova, Phys. Rev. Lett. 113, 146601 (2014).

[21] R. Jansen, A. M. Deac, H. Saito, and S. Yuasa, Phys. Rev. B 85, 134420 (2012).

[22] S. Sato, R. Nakane, and M. Tanaka, Appl. Phys. Lett. 107, 032407 (2015).

[23] T. Uemura, K. Kondo, J. Fujisawa, K.-i. Matsuda, and M. Yamamoto, Appl. Phys. Lett. 101, 132411 (2012).

[24] M. Tran, H. Jaffrès, C. Deranlot, J.-M. George, A. Fert, A. Miard, and A. Lemaître, Phys. Rev. Lett. 102, 036601 (2009).

[25] K. Hipps and U. I. e. t. s. Mazur, Inelastic Electron Tunneling Spectroscopy (Wiley Online Library, 2002).

[26] T. Inokuchi, M. Ishikawa, H. Sugiyama, T. Tanamoto, and Y. Saito, Appl. Phys. Lett. 105, 232401 (2014).

[27] G. Feher, J. Hensel, and E. Gere, Phys. Rev. Lett. 5, 309 (1960).

[28] B. Voisin, R. Maurand, S. Barraud, M. Vinet, X. Jehl, M. Sanquer, J. Renard, and S. De Franceschi, Nano Lett. 16, 88 (2015).

[29] Y. Pu, J. Beardsley, P. M. Odenthal, A. G. Swartz, R. K. Kawakami, P. C. Hammel, E. Johnston-Halperin, J. Sinova, and J. P. Pelz, Appl. Phys. Lett. 103, 012402 (2013).

[30] A. Spiesser, S. Sharma, H. Saito, R. Jansen, S. Yuasa, and K. Ando, Proc. SPIE 8461, 84610K (2012).

[31] T. Sasaki, T. Oikawa, M. Shiraishi, Y. Suzuki, and K. Noguchi, Appl. Phys. Lett. 98, 012508 (2011).

[32] M. Shiraishi, Y. Honda, E. Shikoh, Y. Suzuki, T. Shinjo, T. Sasaki, T. Oikawa, K. Noguchi, and T. Suzuki, Phys. Rev. B 83, 241204 (2011).

[33] Y. Saito, M. Ishikawa, T. Inokuchi, H. Sugiyama, T. Tanamoto, K. Hamaya, and N. Tezuka, IEEE Trans. Magn. 48, 2739 (2012).

[34] A. Fert and H. Jaffres, Phys. Rev. B 64, 184420 (2001).

[35] Y. Song, O. Chalaev, and H. Dery, Phys. Rev. Lett. 113, 167201 (2014).

[36] E. Shikoh, K. Ando, K. Kubo, E. Saitoh, T. Shinjo, and M. Shiraishi, Phys. Rev. Lett. 110, 127201 (2013).

[37] H.-P. Chen, Y. Yuan, B. Yu, J. Ahn, P. C. McIntyre, P. M. Asbeck, M. J. Rodwell, and Y. Taur, IEEE Trans. Electron Devices 59, 2383 (2012). 